\title{
1 Food Safety Education For People with Diabetes and Pregnant Women: a Positive
}

2 Deviance Approach

3

4 Authors: Yaohua Feng ${ }^{1}$,Christine Bruhn ${ }^{1}$, and Health Management and Education ${ }^{2}$,

5 1. Department of Food Science and Technology, University of California, Davis, One Shields

6 Ave, Davis, CA 95616; 2. UC Davis Health System, 4860 Y Street, Sacramento, CA, 95817

7

8 Author correspondence: Christine Bruhn Phone: +1 530.219.2888; Fax: +1 530.752.4759

9 E-mail: cmbruhn@ucdavis.edu

10

11 
14 Few education programs target audiences at high-risk for foodborne illness, especially people with diabetes and pregnant women. The objective of this study is to develop a food safety education program for each using a positive deviance approach. The positive deviance focus

17 group is a novel educational intervention that allows participants to discuss their food handling 18 behaviors and decide to try recommended positive practices modeled by people like

19 themselves. To fit within the educational programs offered by the collaborating institutions, three 20 sessions were given, discussing safe handling topics: 'Cook and Chill,' 'Clean and Separate,' 21 and 'Choose Safe Food.' People with diabetes (32) and pregnant women (29) participated in the 22 study. Focus groups were audiotaped and transcribed. Participants became involved and 23 inspired by vivid discussions and were inspired to change their behaviors to the recommended

24 food handling practices modeled by their peers. Safe food handling practices were reinforced by 25 take-home tasks focused on using refrigerator and cooking thermometers and using separate 26 cutting boards. Participants were unaware of details associated with safe handling

27 recommendations, such as the recommended temperature for cooking specific items or the 28 temperature of the refrigerator. Results of this study indicate that positive deviance discussion 29 module could be a promising alternative to traditional methods of food safety education.

37 Positive deviance; Qualitative; Food safety education; Food handling; High-risk population 
39 Foodborne illness is an important public health issue in the United States. Data collected by the

40 Centers for Disease Control and Prevention's (CDC) reveal a total of 19,056 cases of culture-

41 confirmed bacterial and laboratory-confirmed parasitic infection, 4,200 hospitalizations, and 80

42 deaths among 48 million residents of 10 states, 15\% of the US population (Humes et al., 2011).

43 Some groups are at higher risk for foodborne illness than others. Smith J. L. reported that down-

44 regulation of the cellular immune system induced by hormonal changes during pregnancy

45 increase the risk of pregnant women experiencing foodborne illness (Smith, 1999). Such

46 changes increase the chance of infection from certain foodborne pathogens, like Listeria

47 monocytogenes, Toxoplasma gondii, Brucella species, Salmonella species and Campylobacter

48 jejuni (Szekeres-Bartho, 1990). Pregnant women are considered 17 times more likely than the

49 healthy general population to contract listeriosis from L. monocytogenes (Silver, 1998, Poulsen

50 and Czuprynski, 2013). Certain organisms, including Listeria monocytogenes, Toxoplasma

51 gondii, Salmonella typhi and Campylobacter jejuni, can have adverse consequences for the

52 fetus if they cross placenta (Smith, 1999, Lamont et al., 2011, Poulsen and Czuprynski, 2013).

53 While Escherichia coli O157:H7 and Vibrio cholerae have not been found to cross the placental

54 barrier; they can infect the mother and cause severe consequences for the mother and her fetus

55 (Smith, 1999). Infection can result in miscarriage, stillbirth, premature labor or severe

56 complications for the baby.

57 Diabetes can also increase the risk of foodborne infection (Shah and Hux, 2003). During a

58 Salmonella enteritidis outbreak in which raw eggs were used in a hospital-prepared

59 mayonnaise, patients with diabetes who required insulin or oral hypoglycaemics were at

60 increased risk (Telzak et al., 1991). According to previous research (Calvet and Yoshikawa,

612001 ) campylobacteriosis is four times more common and salmonellosis three times more

62 common in persons with diabetes than in the general population. Goulet and Marchetti (Goulet

63 and Marchetti, 1996) report that persons with diabetes are 25 times more likely to have 
64 listeriosis. The increased risk of infection with Salmonella enteritidis among diabetics is likely

65 due to decreased gastric acid production and decreased bowel motility. The stomach, through

66 the effect of gastric acidity due to hydrochloric acid, functions as a barrier to ingested bacteria

67 (Giannella et al., 1973). At the time of this paper, current literature does not estimate the

68 increased risk of an E. coli infection among pregnant women or people with diabetes.

69 High-risk individuals are generally unaware of their increased vulnerability to foodborne illness.

70 A multi-state survey results showed that less than $20 \%$ of the pregnant women have knowledge

71 about Listeria, which is a crucial pathogen during pregnancy (Ogunmodede et al., 2005). Other

72 studies report that women are unaware of the danger of eating higher risk foods during

73 pregnancy (Athearn et al., 2004, Cates et al., 2004, Trepka et al., 2006). Furthermore, a

74 qualitative study of health care professionals suggests that women are not being educated

75 about this issue during pregnancy (Morales et al., 2004). Few studies address awareness of the

76 increased risk of foodborne illness among people with diabetes. Educational programs for this

77 condition focus on food choice and seldom or never include a discussion of food safety

78 (Institute, 2011).

79 This study was aimed to address the gap of high-risk population food safety education, and to

80 evaluate a food safety educational program which uses a positive deviance approach. Positive

81 deviance is based on the observation that in every community there are certain individuals

82 whose uncommon practices enable them to find better solutions to problems than others despite

83 having access to the same resources. This approach has been successfully used to solve

84 problems in the field of nutrition, like childhood malnutrition (Zeitlin et al., 1993) and poor infant

85 health (Guldan et al., 1993). At the time of this writing, positive deviance has not been used in 86 food safety education. 
METHODS AND MATERIALS

\section{1. Participants}

\subsection{People with diabetes}

90 The authors recruited participants from the University of California Davis Health Center Health

91 Management and Education diabetes classes in Sacramento. The study was announced in 92 classes and study flyers and a blank contact information forms were left with participants. The

93 interested parties could contact the author by phone or email. All participants were required to

94 be 18 years old or older, have Type I or II diabetes, act as the primary meal preparer in their

95 family, and cook meals at least three times a week. Classes were scheduled based upon

96 participant's availability. After four months of recruitment (from January 2014 to May 2014), 32

97 people with diabetes were recruited (30 completed all three sessions and the post-survey).

98 Participants were separated into six groups of four to eight people each.

\subsection{Pregnant women}

101 'centering program meeting', and they were grouped by their due date months, for example, the 102 participants, whose due dates were in May, were grouped together. The author announced the 103 study in their centering program meetings, and handed out the consent forms and pre-surveys

104 to those who indicated that they would like to participate in the study. Participants from Women

105 Infants and Children (WIC) were recruited through flyers in the office lobby. Inclusion criteria

106 stated that participants must prepare meals at least three times per week, and act as the

107 primary meal preparer in the family. Discussions with participants from Sutter Health Davis took

108 place in a conference room in the Sutter Health Davis hospital, and with participants from WIC

109 took place in a conference room in UC Davis Food Science department. Participants were

110 advised that they could discontinue participating in the study at any time. In return for

111 participation, participants were given a $\$ 30$ gift card. The study was approved through the

112 Institutional Review Board, University of California, Davis. 


\section{Procedure}

114 At the first meeting all participants completed a survey, which measured their safe food handling

115 knowledge, and perceived risk of contracting foodborne illness. The pre-survey included four

116 self-efficacy questions that were adapted from previous work (Byrd-Bredbenner et al., 2007);

117 two self-risk assessment questions developed by the authors; and eleven food safety

118 knowledge questions that were adapted from an educational program delivered in California

119 (Blackburn et al., 2014). At the conclusion of the food safety intervention, participants completed

120 a post-survey which consisted of three program evaluation questions and the same self-risk

121 assessment and food safety knowledge questions in the pre-survey.

122 To fit within the education programs cooperated by the medical institutions, three PD group

123 sessions were led by the authors. Due to the nature of the programs for the two audiences,

124 each PD session with the diabetes group lasted one and one-half hours; while the pregnant

125 women sessions were one hour. Topics for each session were 'Cook and Chill', 'Clean and

126 Separate' and 'Choose Safe Food'. Topics were selected based on the educational programs

127 offered by the Partnership for Food Safety (www.fightbac.org). Previous research (Anderson et

128 al., 2011, Kosa et al., 2015, Nesbitt et al., 2014)also showed that consumers especially need

129 information around those topics. In each session the author provided an introduction, answered

130 questions and conducted a structured discussion using a written guide (Error! Reference

131 source not found.) based on the Positive Deviance approach (Zeitlin et al., 1993). Participants

132 were invited to discuss their food handling practices related to the topic. If a respondent was

133 practicing the recommended behavior, the group would analyze the strategy leading to this

134 behavior. The discussion would then lead to a consensus that others would try the behavior

135 before the next session. Finally the entire group was assigned a take home task that reinforced

136 the concepts discussed. A co-moderator took notes and operated the tape recorder. After the

137 first session, the participants were provided a cooking thermometer (model IRT220, Component

138 Design Northwest, Inc., Portland, OR), a refrigerator thermometer (model FG80, Component 
Design Northwest, Inc., Portland, OR), and a take-home task sheet (see Error! Reference source not found.). After the second session, the participants were provided three cutting mats

141 (Greenbrier International, Inc., Chesapeake, VA), and a take-home task sheet (see Error!

142 Reference source not found.). After the third session, post-survey data was collected. A

143 follow-up telephone interview took place two to three weeks after the third session. Employing a 144 casual chatty manner, the author asked

145 What did you think about the program?

146 Do you do anything differently now after completing the program?

147 Audio recording with notes were taken during the session by an assistant. All sessions were 148 audio-taped, transcribed verbatim, reviewed for accuracy, and entered into and analyzed using

149 XSight (version 2.0.75.0 SP2, QSR International, Cambridge, MA), a qualitative data

150 management software program. All procedures were pilot tested with a group of 6 participants

151 prior to finalizing the questions and recruiting the study population. The University of California,

152 Davis's Institutional Review Board approved this study.

\section{RESULTS}

\section{1. Demographic}

155 Participants consisted of thirty people with diabetes, with $57 \%$ females. See Table 1 . The

156 majority, $60 \%$, was above 60 years old. Most participants identified as of Caucasian decent

157 (73\%), 13\% as Hispanic, 10\% identified as Asian descent, and 3\% identified with African-

158 American decent. For the pregnant women group $(n=29), 55 \%$ were in age group 20-29, and

$15945 \%$ were between 30 and 39. Most of the participants identified as of Caucasian decent (69\%),

160 followed by $28 \%$ Hispanics,

161 2. Session I: 'Chill' and 'Cook'

162 The focus of the first session was temperature control. Participants were invited to discuss the 163 issues related to 'chill', including 'thawing food', 'storage of leftovers', 'storage of groceries' and 164 'keeping refrigerator at recommended temperature'. In response to the probe, 'What are some 
165 of the things you do to keep the temperature of the refrigerator cool enough,' some participants

166 responded that they used a thermometer, and others stated that they would like to try this

167 practice.

168 The participants also discussed the issues related to 'cook'. 'How do you tell when your food is

169 done?' was asked, and at least one participant in every group mentioned that they used a

170 cooking thermometer. The author then led the discussion to focus on the merits or

171 disadvantages of using a thermometer. Following this, the author led the participants toward a

172 consensus to practice using a cooking thermometer.

173 At the conclusion of the session, each participant was given a refrigerator and cooking

174 thermometer. They were asked to complete two "homework" assignments: record the

175 temperature in at least three places in their refrigerator and record the final temperature of at

176 least two items they either cooked or, if leftovers, warmed up. These take-home tasks were

177 selected to reinforce the concepts discussed and provide an opportunity for participants to

178 practice the recommended behaviors.

$179 \quad 2.1$ Comments related to chilling food

$180 \quad$ 2.1.1 Refrigerator Temperature

181 Most participants did not know their refrigerator temperature. Some participants had

182 refrigerators with a built-in thermometer, however, this thermometer only measures one area of

183 the refrigerator. That the temperature on the built-in thermometer was not accurate for every

184 part of the refrigerator, was a surprise for many participants. Participants noticed that different

185 parts of the refrigerator had different temperatures, and usually the closer to the freezer or the

186 main cooling fan, the colder the area. The shelf in the refrigerator door was the warmest area of 187 the refrigerator.

My fridge tells me but I don't trust it. I bought one to tell me. And especially the door, I want to make sure my milk is safe because I leave it on the door. (Pregnant women) 
Our fridge has numbers 1-9, I just make sure foods in front are not frozen. (Diabetics)

191 Some participants organized their refrigerators by temperature, not according to the

192 recommendation to store raw meat below cooked ready-to-eat food.

I put leftover on the bottom shelf, because that is the coldest. (Diabetics)

I was surprised because I keep my eggs by the freezer sides and I moved it to the other side because it started looking weird and it was even colder. (Pregnant women)

\subsubsection{Thawing Food}

198 the refrigerator. During the discussion about strategies to comply with this practice, many

199 participants mentioned they would follow 'alternative' methods if they needed the meat sooner.

200 However those 'alternative' methods were usually not recommended, like putting the item in

201 sink, on the counter, or in the sun. After the discussion, all participants said they would try to

202 thaw meat in the refrigerator.

\section{2.1.3 Take-home task}

204 The first take-home task was to measure the temperature of different spots of their home

205 refrigerators using a refrigerator thermometer. Before completing this task, only one out of thirty-

206 four pregnant women had a separate refrigerator thermometer at home, and about one third had

207 a built-in thermometer in their refrigerator. Only three of participants recalled the temperature of

208 their refrigerator. Among diabetic participants, $20 \%$ had a built-in thermometer in their

209 refrigerator. Over half were not sure of the recommended refrigerator temperature.

210 Twenty-five out of thirty participants in the diabetics group and twenty out of twenty nine

211 pregnant women turned in their take-home task report sheets of refrigerator temperatures, and

212 the participants who did not turn in the report sheets claimed that they also completed the take

213 home tasks. One third of participants from the diabetics group and over $40 \%$ from pregnant

214 women group reported refrigerator top-shelf temperatures higher than $40^{\circ} \mathrm{F}$ (Error! Reference 
215 source not found.). All participants reported the temperature at the refrigerator door was the 216 highest.

\section{$217 \quad 2.2$ Comments related to cooking food}

218 Most of the participants were confident as to their cooking skills. When asked how they knew

219 when the poultry or burgers was cooked, they all agreed that 'white' or 'no pink' was the

220 indicator for chicken parts, and some used 'color' and some used 'touching, feel' for burger. The

221 only time most of them mentioned using thermometer was cooking whole poultry. Some

222 participants did not have thermometers, and they used a 'pop-up timer' which came with the

223 turkey package. They did not know what temperature the 'pop-up timer' was set to.

When my family makes whole turkey, I eat the dark meat. We strictly tell by color not by temperature. (Diabetes)

I usually go with look and color. Only time we use a thermometer is for roast turkey. (Pregnant woman)

\subsubsection{Using a cooking thermometer}

The participants asked many questions about using a thermometer, which reflected some of the 230 reasons why they did not use the thermometers frequently. First, they did not know the 231 recommended temperature for different cooking items;

What is the temperature for chicken? Is that $180^{\circ} \mathrm{F}$ or $185^{\circ} \mathrm{F}$ ? (Pregnant women)

Should I cook my leftovers to the same temperature $\left(165^{\circ} \mathrm{F}\right)$ ? (Diabetes)

234 Secondly, they did not know how to use the thermometer, when cooking small or thin pieces;

It's so small that you just poke through the whole thing. (Diabetes)

236 Thirdly, they never learned the thermometer should be calibrated or how to calibrate it. Since an 237 inaccurate thermometer could result in overcooked or undercooked food items the thermometer 238 was viewed as less reliable than appearance and texture; 

women)

241 Many of the participants did not know why it is important to cook the food to certain temperature.

242 Some mentioned that they just followed the recipe or the cooking show.

Why not the same temperature for everything? It would be much easier for us to remember. (Pregnant women)

Some participants said they trust the meat quality, so it would not be necessary to cook it to that high temperature: quality burger at the market then it's not safe. (Diabetes)

252 the thermometer, and they agreed that it was not as difficult as they expected. Some said they 253 would use a thermometer more often in the future, however some still struggled with using the 254 thermometer. I could not get the temperature up to the recommended temperatures for a microwave meal. (Pregnant women)

but most participants enjoyed the experience:

262 what they should do to keep them safe. When asked how to reheat leftovers, many participants

263 didn't know the recommended end point temperature for leftovers. Most used a microwave to 264 reheat the items until steamy, and only used the oven for baked food like pizza. 


\subsubsection{Take-home task}

The first take-home task also included measuring the temperature of home cooked items using

267 a cooking thermometer. Among pregnant women $60 \%$ had cooking thermometer at home, and

268 most only used the thermometer when cooking whole poultry. Half of the persons with diabetes

269 had a cooking thermometer at home, and all only used it when cooking whole poultry.

270 Participants were asked to record the end point temperature of three items. About $80 \%$ of the

271 cooked items from the diabetics participants, and two third of the cooking items from the

272 pregnant women were at or above the recommended temperature. The items not reaching the

273 recommended temperature, were either because the participants did not remember the

274 recommended temperature or because of misuse of the thermometer. Four participants in the

275 diabetics group and two in the pregnant women group reported their thermometer could not

276 reach the recommended higher temperature, even though they kept cooking the food items.

\section{3. Session II: 'Clean' and 'Separate'}

278 During the second session, participants were led to discuss issues related to 'clean' and

279 'separate', including 'washing hands', 'washing fruit and vegetable', 'avoiding washing poultry

280 under running water' and avoiding 'cross-contamination'. In response to the probe, 'What are

281 some of the things you do to avoid cross contamination?' some participants responded using

282 separate cutting boards and cleaning kitchen items that were highly likely to contribute to get

283 cross contamination. Others indicated that they would like to try to practice these behaviors.

284 After the session, each participant was given two cutting boards and asked to use a separate

285 cutting board for items to be eating raw, such as fruit or greens, and items to be cooked, like

286 raw meat, poultry, or fish. They were also asked to record how frequently they cleaned specific

287 kitchen items: sink, counter, utensils, cutting boards.

$288 \quad 3.1$ Comments about 'Clean'

289 'Clean' was a more familiar topic for all participants. They immediately linked 'clean' to hand 290 washing and produce washing. 
292 Almost all participants knew they should use soap and water to wash hands, and they

293 responded with various versions for duration of washing:

I use the hottest water I can, and use soap, and I sing happy birthday. (Pregnant woman)

However, when asked how long they actually washed their hands in daily life, the washing duration was much shorter:

A lot of people know 20s but they don't do it. I am one of them. (Diabetes)

I usually don't pay attention until the soap is off my hand and that's it. (Pregnant woman)

Some participants just did not believe their hands were 'that dirty'.

If I am at home all the time, do I still need to wash my hands that often? (Diabetes)

\subsubsection{Washing fruit and vegetable}

Most participants reported that they washed their vegetables before eating. One participant said she had never washed an apple before. She thought everything she bought from the supermarket was free of germs. Many participants did not wash fruit that needed to be peeled, like oranges, watermelon, and pineapple. After the explanation that cutting through a fruit would transfer germs from the outside to the inside, participants were much more comfortable with complying with the recommendation to wash all fruit.

311 scientific documentation, such as using a little vinegar or salt in the wash water.

312 If strawberries, I also put vinegar in the water and soak them for 5-10 minutes. (Pregnant 313 women)

$314 \quad$ What if you do a vinegar wash like my mother-in-law to keep it fresh longer? (Diabetics) 
315 When asked whether they wash the 'pre-washed' salad leaves, almost half of the participants

316 did not wash these products. Many who still wash them indicated that they did not trust the

317 manufactories or merchants to wash adequately.

$318 \quad$ 3.1.3 Take-home task

319 The second take-home task for this session was to record the frequency of kitchen items

320 cleaning. Twenty-three out of thirty participants in the diabetics group and nineteen out of twenty

321 nine participants in the pregnant women group turned in their take-home task report sheets

322 recording usage of multiple cutting boards and frequency of cleaning home kitchen items.

323 Participants who did not turn in the report sheets claimed that they also completed the take

324 home tasks.

325 The frequency of cleaning activities in a week among both people with diabetes and pregnant

326 women is presented in Figure 1. Participants cleaned their utensils and cutting boards much

327 more frequently than their sinks and countertops. Before this take-home task, some participants

328 seldom cleaned their sink. Participants commented that understanding the 'cross-contamination'

329 concept, caused them to pay more attention to cleaning other items in their kitchen such as the

330 countertop and sink.

\subsection{Comments about 'Separate'}

332 Cross contamination was introduced into the focus groups as part of the discussion around

333 'Separate'. This was the first time most participants had heard about this concept. However,

334 after introducing this word, it was frequently used by participants in later discussions.

$335 \quad$ 3.2.1 Cleaning in the home kitchen (avoiding cross-contamination)

336 Participants agreed that using a dishwasher was a good way to sanitize the utensils and cutting

337 board in the home kitchen because of the high heat used by the washer. However, half of the

338 participants did not have a dishwasher at home. Many participants used sponges or wash

339 clothes to wash utensils and other cooking ware, but two focus groups suggested using a 
340 microwave to sanitize the sponge and using a washing machine to sanitize a wash cloth. Most

341 participants did not consider a sponge or wash cloth as a medium of cross contamination.

342 After the take-home task to record cleaning items in their kitchen, all participants agreed that

343 they should clean their countertop and sink more often. Participants mentioned many other

344 kitchen tools that were not listed on the take-home task, which suggests that the take-home task

345 raised their food safety alertness.

346 3.2.2 Separating cutting board (avoiding cross-contamination)

347 Most participants agreed that using a separate cutting board for raw meat and fruit and

348 vegetable was an appropriate safe food handling practice. It was common to have more than

349 one cutting board at home.

$350 \quad$ I bought a glass cutting boards that goes in the dishwasher. I have been using those especially for meat so I wouldn't use the same one for raw meat. I believe they should sterilize well in the dishwasher. (Diabetes)

When asked how they used their cutting boards, many the participants responded they used a

354 different board for meat and produce. Some participants, more people with diabetes than

355 pregnant women, used only one cutting board for everything. They did this out of convenience

356 and believed that 'rinsing between usages' (without soap) was sufficient. My boyfriend will cut everything on the same board but I tell him to rinse with soap and

\subsubsection{Take-home task}

362 After completing the second take-home task which specified using separate cutting boards, all

363 participants reported they separated the cutting boards for raw meat and fruit. One of the

364 participants who was a vegetarian used a separate board for fruit and vegetables. 


\section{Session III: 'Choose'}

During the third session, the participants discussed issues related to 'choose safe food', including 'choosing pasteurized juice/milk/soft cheese', 'sell-by and use-by date', and 'food irradiation'. When participants were asked, 'Do you check if the juice and milk you buy is pasteurized', some participants responded positive on both, and the others also agreed this was a practice that was feasible in their daily life. Since this was the last session, no take home tasks were assigned.

\subsection{Comments about choose safe food}

Diabetics participants were more engaged in this topics than pregnant women. Some even took notes with them about the food items that were safe or less safe to eat. Not eating runny yolk egg and cookie dough were the two practices most difficult for participants to adopt.

Over easy eggs? I always eat my eggs over easy. (Diabetes)

I have thought about it (cookie dough) with the raw eggs. I do think about it briefly but it doesn't stop me from licking off my fingers or something. (Pregnant women)

Many pregnant participants were surprised that deli meat may contain Listeria. Some of them said, 'if it is not safe, then I will not eat it for the rest of my pregnancy."

\subsection{Comments about food irradiation}

When told that irradiation would make food safer by destroying harmful bacteria, persons with diabetes were much more open to the idea of irradiated food compared to the pregnant women. Around two thirds of the diabetic participants responded that they would like to 'try' irradiated food, if it is safer to eat. In contrast, only one third of the participants in the pregnant women group said they would like to try irradiated food. Those who were hesitant to try said they had not seen evidence that irradiated foods are safe.

I won't eat it. It is just too new to me. (Pregnant woman) I would rather not eat meat than eat irradiated (meat). (Pregnant woman) 
Although not part of the topics covered, pregnant women also raised many questions about controversy topics, like genetically modified food. Most of them were negative about what they

392 perceived as "unnatural concepts" in food.

393

394

395

396

397

398

When asked 'do you do anything differently after the program?' many participants in both

\section{Comments about this program}

The program was well received by the participants in both the diabetes and the pregnant women groups. Many described the facts delivered in the program as 'surprising' and 'memorable'. All participants agreed the information in the program was helpful to them and would recommend the program to other people with the same condition, diabetes and pregnancy.

The info about the luncheon meat is surprising. These are eye openers. (Diabetes) However, there were also complaints about this program, mainly from pregnant women, some felt stressed with all the new information they had received.

Now, I feel I am a bit paranoid with the food I eat. (Pregnant woman)

\section{Follow-up evaluation}

Twenty-seven of thirty participants with diabetes and fourteen out of twenty nine pregnant women participated in the follow-up interview. The low rate of pregnant women group participation was mainly because the pregnant women had not previously received a phone call from the author, while participants in diabetics group received the author's phone call many times for scheduling and reminding of the sessions. Additionally when the follow up call was scheduled, it was difficult for the new moms to find time in their schedule to talk.

When asked 'what do you think about the program', participants with diabetes were still very excited about the information they had received. Most pregnant women said it was helpful, and some said they would still prefer more information on childbirth, which was their primary focus. groups mentioned using refrigerator and cooking thermometers. Some also reported that they were more careful about hand washing and home kitchen cleaning. 
418 Many people with diabetes and pregnant women did not know they were at increased risk of

419 foodborne illness. This is consisted with previous studies (Athearn et al., 2004, Cates et al.,

420 2004, Trepka et al., 2006, Ogunmodede et al., 2005). Even if participants were aware that

421 people could become ill from food handling mistakes, and they knew what was recommended,

422 like washing hands for 20 s, they still would not do it every time. Consumers tended to associate

423 foodborne illness with food handling violations at restaurants instead of home cooking. Others

424 have also reported that consumers perceive home produced food as lower risk, and food

425 prepared by others as higher risk (Frewer et al., 1994, Bruhn, 2014).

426 The above phenomena may be due to 'Optimism Bias,' in which people perceive their own risk

427 of getting foodborne illness or handling food unsafely is lower than that of others. 'Optimism

428 Bias' can reduce the consumer's health-protective behaviors and their precautions regarding

429 personal risks (da Cunha et al., 2014). Optimistic bias is not unique to consumers in their home.

430 A recent study in Brazil (da Cunha et al., 2014) found that food handlers perceived themselves

431 as less likely than their peers to transmit a foodborne disease to consumer and their friends.

432 Differences in session involvement were found between pregnant women and people with

433 diabetes. Pregnant women tended to have a lower turn-in rate of take-home task response and

434 were less involved in the focus group discussions. This difference could be related to medical

435 condition length (pregnant women facing short-term condition vs. people with diabetes facing

436 long-term condition), recruitment (pregnant women from university community vs people with

437 diabetes from metropolitan area), age-group (pregnant women in 20-39 age group vs. people

438 with diabetes in 40-79 age group), and motivation (pregnant women focused childbirth vs

439 people with diabetes focused on improving diet and health). People with diabetes were older,

440 and were more comfortable accepting recommendations from health authorities. Those with 
441 diabetes were from metropolitan area, and were less skeptical about information from a

442 university source. Additionally, those with diabetes were motivated to improve their health.

443 Also, when talking about food irradiation, pregnant women were less open to this process than

444 people with diabetes. Participants also wondered if irradiation affects tastes nutrition, and

445 safety. Since irradiated food is not widely available in the marketplace, few had a chance to

446 personally verify the sensory qualities of irradiated items. Previous research found that

447 insufficient information about the risks, benefits and safety concerns are major factors driving

448 consumers reluctance of buying irradiated food (Fox et al., 2001, Fox et al., 2002, Boland and

449 Fox, 2012).

450 To reach the target audiences, food safety educators must gain the support of health educators

451 currently offering pregnancy or nutrition management programs. To recruit a larger audience,

452 food safety educators should consider adding food safety education to existing programs

453 compared to offering food safety education alone. Mentioning surprising food safety information

454 during recruitment motivates the audience to attend. Props can help dramatize facts on bacteria

455 multiplication or cross contamination. Take-home tasks enable the participant to practice

456 recommended behavior often with surprising results which can motive further compliance to

457 recommendations. The video about chicken washing allowed participants to visualize cross

458 contamination making the concept much more easily understood.

459 Participants frequently asked why a specific action should be taken. Many times, they were 460 surprised by the reason behind a specific recommendation. For example, they wanted to know 461 why the cooking end point temperature for poultry was higher than that for ground beef. When 462 they heard that Salmonella requires a higher killing temperature than E. coli. they understood 463 the rational for the difference and were also reminded that these healthy foods could contain 464 pathogens that required control. While brief and succinct recommendations are more easily 
communicated, this research suggests that a short explanation may assist consumers in

466 remembering and following the guidelines.

467 A discrepancy in poultry recommended end-point cooking temperature led to consumer

468 confusion. The author found that there were recipes in cook books, web sources, and blogs that

469 still recommended $185^{\circ} \mathrm{F}$ as the end point temperature. Further, some cooking thermometers

470 currently for sale in the supermarket listed on the dial $185^{\circ} \mathrm{F}$ as the recommended temperature

471 for poultry. It is necessary for book and blog publishers and thermometer manufacturers to

472 update their products' temperature recommendation to $165^{\circ} \mathrm{F}$.

473 The results from this study indicate that a positive deviance discussion module could be a

474 promising alternative to traditional methods of food safety education. Although traditional food

475 safety reading materials can increase food safety knowledge, consumers could be more

476 involved and inspired by vivid discussions. Participants indicated that they changed their

477 behavior to the recommended handling practice, by following their peers' positive deviance.

478 Further, the discussion format provides insight into the current knowledge of participants and

479 the living condition challenges they face. This can guide educators in shaping and delivering a

480 more effective food safety program. For example, that women pregnant with their first child were

481 seeking information and those who had previously delivered believed they were well informed

482 suggests that first time moms should be a higher priority in program delivery.

483 A curriculum for health professionals to deliver to high-risk populations is being developed

484 based upon this work. Increased emphasis on food safety education by hospital associated

485 health professionals will help protect people at increased risk of foodborne illness from

486 infections and resulting complications such as miscarriages, reactive arthritis and Guillain Barre'

487 syndrome. Future positive deviance food safety projects should include additional high-risk

488 population groups, like people with compromised immune systems. 
489 One of the limitations of this study was that the results were based on self-report data.

490 Consumers were overly optimistic about their food handling practices, and over report their

491 compliance with safe handling recommendations, compared to actual observed practices

492 (Bruhn, 2014, Phang and Bruhn, 2011). Future research should expand the evaluation of

493 educational interventions to include observational research.

\section{ACKNOWLEDGEMENTS}

495 We thank Sutter Davis Women's Health Midwives' Group, especially Blanche Skubic, Jessica

496 Nagel, and Jennifer Taylor; Yolo County WIC (Women, Infant and Children) program; and UC

497 Davis Health System OBGYN group. This material is based upon work that is supported by the

498 National Institute of Food and Agriculture, U.S. Department of Agriculture, under award number 499 2012-68003-30155.

\section{REFERENCE}

501 Anderson, A. L., Verrill, L. A. \& Sahyoun, N. R. (2011), "Food Safety Perceptions and Practices

502 of Older Adults", Public Health Reports, Vol. 126 No. 2, pp. 220-227.

503 Athearn, P. N., Kendall, P. A., Hillers, V. V., Schroeder, M., Bergmann, V., Chen, G. \&

504 Medeiros, L. C. (2004), "Awareness and acceptance of current food safety

505 recommendations during pregnancy", Maternal and Child Health Journal, Vol. 8 No. 3 ,

$506 \quad$ pp. 149-162.

507 Blackburn, M. L., Bruhn, C. M., Miller, L. S., Ganthavorn, C. \& Ober, B. (2014), "Seniors, and

508 their food handlers and caregivers, need food safety and nutrition education", California

$509 \quad$ Agriculture, Vol. 68 No. 1, pp. 30-37.

510 Boland, M. \& Fox, S. 2012. Food irradiation and public health. Available: Brief Irradiation UDC 2013.pdf [Accessed 12 October, 2015].

513 Bruhn, C. M. (2014), "Chicken preparation in the home: An observational study", Food Prot

514 Trends, Vol. 34 No. 5, pp. 318-330. 
515 Byrd-Bredbenner, C., Wheatley, V., Schaffner, D., Bruhn, C., Blalock, L. \& Maurer, J. (2007), "Development of food safety psychosocial questionnaires for young adults", Journal of Food Science Education, Vol. 6 No. 2, pp. 30-37.

518 Calvet, H. M. \& Yoshikawa, T. T. (2001), "Infections in diabetes", Infectious Disease Clinics of North America, Vol. 15 No. 2, pp. 407-421.

Cates, S. C., Carter-Young, H. L., Conley, S. \& O'brien, B. (2004), "Pregnant women and listeriosis: preferred educational messages and delivery mechanisms", Journal of Nutrition Education and Behavior, Vol. 36 No. 3, pp. 121-127.

Da Cunha, D. T., Stedefeldt, E. \& De Rosso, V. V. (2014), "He is worse than I am: The positive

Fox, J., Bruhn, C. \& Sapp, S. (2001), Consumer acceptance of irradiated meats. In: HOOKER, N. \& MURANO, E. (eds.) Interdisciplinary food safety research. CRC Press, Boca Raton, FL.

Fox, J. A., Hayes, D. J. \& Shogren, J. F. (2002), "Consumer preferences for food irradiation: outlook of food handlers about foodborne disease", Food Quality and Preference, Vol. 35 No. 0, pp. 95-97. How favorable and unfavorable descriptions affect preferences for irradiated pork in experimental auctions", J. Risk Uncertain, Vol. 24 No. 1, pp. 75-95.

Frewer, L. J., Shepherd, R. \& Sparks, P. (1994), "The interrelationship between perceived knowledge, control and risk associated with a range of food-related hazards targeted at the individual, other people and society", Journal of Food Safety, Vol. 14 No. 1, pp. 1940.

Giannella, R. A., Broitman, S. A. \& Zamcheck, N. (1973), "Influence of gastric acidity on bacterial and parasitic enteric infectionsa perspective", Annals of Internal Medicine, Vol. 78 No. 2, pp. 271-276. 
539

Goulet, V. \& Marchetti, P. (1996), "Listeriosis in 225 non-pregnant patients in 1992: Clinical aspects and outcome in relation to predisposing conditions", Scandinavian Journal of Infectious Diseases, Vol. 28 No. 4, pp. 367-374.

Guldan, G. S., Zhang, M. Y., Zhang, Y. P., Hong, J. R., Zhang, H. X., Fu, S. Y. \& Fu, N. S. (1993), "Weaning practices and growth in rural sichuan infants - a positive deviance study", Journal of Tropical Pediatrics, Vol. 39 No. 3, pp. 168-175.

Humes, K., Jones, N. A. \& Ramirez, R. R. (2011), "Overview of race and Hispanic origin, 2010", US Department of Commerce, Economics and Statistics Administration, US Census Bureau.

Institute, T. P. E. 2011. Diabetes and Meal Planning.

Kosa, K. M., Cates, S. C., Bradley, S., Chambers, I. \& Godwin, S. (2015), "Consumer-Reported Handling of Raw Poultry Products at Home: Results from a National Survey", Journal of Food Protection $\AA$, Vol. 78 No. 1, pp. 180-186.

Lamont, R. F., Sobel, J., Mazaki-Tovi, S., Kusanovic, J. P., Vaisbuch, E., Kim, S. K., Uldbjerg, N. \& Romero, R. (2011), "Listeriosis in human pregnancy: a systematic review", Journal of perinatal medicine, Vol. 39 No. 3, pp. 227-236.

Morales, S., Kendall, P. A., Medeiros, L. C., Hillers, V. \& Schroeder, M. (2004), "Health care providers' attitudes toward current food safety recommendations for pregnant women", Applied Nursing Research, Vol. 17 No. 3, pp. 178-186.

Nesbitt, A., Thomas, M. K., Marshall, B., Snedeker, K., Meleta, K., Watson, B. \& Bienefeld, M. (2014), "Baseline for consumer food safety knowledge and behaviour in Canada", Food Control, Vol. 38, pp. 157-173.

Ogunmodede, F., Jones, J. L., Scheftel, J., Kirkland, E., Schulkin, J. \& Lynfield, R. (2005), "Listeriosis prevention knowledge among pregnant women in the USA", Infectious Diseases in Obstetrics and Gynecology, Vol. 13 No. 1, pp. 11-15. 
564 Phang, H. S. \& Bruhn, C. M. (2011), "Burger preparation: What consumers say and do in their home", J. Food Prot., Vol. 74 No. 10, pp. 1708-1716.

566 Poulsen, K. P. \& Czuprynski, C. J. (2013), "Pathogenesis of listeriosis during pregnancy", $567 \quad$ Animal Health Research Reviews, Vol. 14 No. 01, pp. 30-39.

568 Shah, B. R. \& Hux, J. E. (2003), "Quantifying the risk of infectious diseases for people with 569 diabetes", Diabetes Care, Vol. 26 No. 2, pp. 510-513.

570 Silver, H. M. (1998), "Listeriosis during pregnancy", Obstet Gynecol Surv, Vol. 53 No. 12, pp. $571 \quad 737-40$.

572 Smith, J. L. (1999), "Foodborne Infections during Pregnancy", Journal of Food Protection, Vol. $573 \quad 62$, pp. 818-829.

574 Szekeres-Bartho, J. (1990), "Endocrine regulation of the immune system during pregnancy", $575 \quad$ Arch Immunol Ther Exp (Warsz), Vol. 38 No. 1-2, pp. 125-40.

576 Telzak, E. E., Greenberg, M. S. Z., Budnick, L. D., Singh, T. \& Blum, S. (1991), "Diabetes577 mellitus - a newly described risk factor for infection from Salmonella-Enteritidis", Journal $578 \quad$ of Infectious Diseases, Vol. 164 No. 3, pp. 538-541.

579 Trepka, M. J., Murunga, V., Cherry, S., Huffman, F. G. \& Dixon, Z. (2006), "Food safety beliefs 580 and barriers to safe food handling among WIC program clients, Miami, Florida", Journal $581 \quad$ of nutrition education and behavior, Vol. 38 No. 6, pp. 371-377.

582 Zeitlin, M., Ghassemi, H. \& Mansour, M. (1993), "Positive deviance in child nutrition - a $583 \quad$ discussion", Ecology of Food and Nutrition, Vol. 30 No. 2, pp. 79-87. 
1 Table 1 Demographic information of study participants

\begin{tabular}{ccc}
\hline & $\begin{array}{c}\text { Diabetics } \\
\mathrm{n}=30 \\
\%(\mathrm{n})\end{array}$ & $\begin{array}{c}\text { Pregnancy } \\
\mathrm{n}=29 \\
\%(\mathrm{n})\end{array}$ \\
\hline Gender & & \\
Male & $43(13)$ & 0 \\
Female & $57(17)$ & $100(29)$ \\
Age group & & \\
$20-29$ & 0 & $55(16)$ \\
$30-39$ & 0 & $45(13)$ \\
$40-49$ & $13(4)$ & 0 \\
$50-59$ & $27(8)$ & 0 \\
$60-69$ & $47(14)$ & 0 \\
$70-79$ & $13(4)$ & 0 \\
Ethnicity & & \\
Caucasian & $73(22)$ & $69(20)$ \\
Hispanic & $13(4)$ & $28(8)$ \\
Asian & $10(3)$ & $3(1)$ \\
African-American & $3(1)$ & 0 \\
\hline
\end{tabular}

2

3 


\section{Session 1}

\section{Background}

What is foodborne illness?

Who do you think are high-risk to foodborne illness?

How much risk do you think you are after you have diabetes (or you are pregnant), compared with general population?

What foods do you think are commonly associated with foodborne illness?

Have you heard of safety issues relate to food? If so, what have you heard of?

\section{Chill}

How long should left-overs be at room temperature before going to refrigerator?

How far are you from your grocery or favorite to-go restaurant?

When do you put leftovers in the refrigerator? What kind of container do you use to put them in the refrigerator?

Do you feel comfortable in your own home that foods are kept cold enough?

If yes, tell us what you do

If no, what exactly are your concerns?

Do you know how cold your refrigerator is? Do you know how cold it should be?

What are some of the things you do to make sure the refrigerator to stay cold? (PD)

\section{Cook}

How do you tell when your food is done?

Have you ever thought food was done, and then you cut into it, the food was only

lukewarm? If this has never happened to you, what is your secret? (PD)

Do you ever use a food thermometer? Why or Why not?

\section{Session 2}

\section{Checking Take-home task I}

How did you managed the cooking and chilling projects? Did everything go well? Let's talk about the results.

\section{Clean}

When and how to wash hands

How often do you think you actually wash your hands?

Are you concerned about the safety of the produce that you purchase? Why or why not?

Do you wash poultry under running water?

Do you think everyone does a good job in cleaning?

What are some of the things you do to be sure the food and the kitchen are clean? (PD)

\section{Separate}

Do you remember seeing these 'cross-contamination' mistakes?

What are some of the things you do to avoid cross contamination?(PD) 


\section{Session 3}

\section{Checking Take-home task II}

What did you do and how did it work for you?

\section{Choose}

Are you concerned about the safety of the produce that you purchase? Why or why not? Have you heard of any problems associated with drinking raw milk or un-pasteurized juice? If yes, what have you heard?

Do you buy raw milk, juice or soft un-ripened cheese with raw milk? Why or why not? (PD) Have you heard people getting ill from soft un-ripened cheese, like Brie or soft Mexican cheese (Queso Fresco).

Do you know any strategy to make these foods safe? (PD) Have you heard of any meat recalls or people becoming ill from eating burgers? If yes, what have you heard?

Do you check dates on the perishable foods you purchase from the market?

Do you know the difference between 'best-use-by-date' and 'use-by-date'?

What are some of the things you can do to reduce your risk?

Have heard of irradiation? Have you tried irradiated meat? Would you like to? 
8 Table 3 Take-home task response sheet - Task I

$\begin{array}{ll}\text { Name: } & \\ \text { Refrigerator Thermometer } & \text { Door__ } \\ & \text { Top shelf } \\ & \text { Bottom shelf } \\ & \text { Other }\end{array}$

Food thermometer

\begin{tabular}{l|l} 
Item & Temperature
\end{tabular}

9 
11 Table 4 Take-home task response sheet - Task II

\section{Name:}

How do you use those two cutting boards? (separate or mixed)

\section{Cleaning checkbox}

\begin{tabular}{||l|l|l|}
\hline Item & $\begin{array}{l}\text { How many times you } \\
\text { cleaned in this week }\end{array}$ & $\begin{array}{l}\text { How you cleaned (paper } \\
\text { towel or cloth) }\end{array}$ \\
\hline Utensils & & \\
\hline Countertop & & \\
\hline Sink & & \\
\hline Cutting boards & & \\
\hline
\end{tabular}

12

13 
14 Table 5 Refrigerator temperature locations that were higher than $40^{\circ} \mathrm{F}$

\begin{tabular}{lcc}
\hline & People with diabetes & Pregnant women \\
& $\mathrm{N}=25$ & $\mathrm{~N}=20$ \\
$\%(\mathrm{n})$ & $\%(\mathrm{n})$ \\
\hline Door & $39(10)$ & $45(9)$ \\
Top-shelf & $29(7)$ & $41(8)$ \\
Bottom-shelf & $32(8)$ & $35(7)$ \\
\hline
\end{tabular}

15 
16 Table 6 Most common volunteered behavior that changed after completing the program

\begin{tabular}{lc}
\hline \multicolumn{1}{c}{ Item } & $\begin{array}{c}\text { Total } \\
\text { Sample }\end{array}$ \\
& $\mathrm{N}=41$ \\
& $\%(\mathrm{n})$ \\
\hline Use a cooking thermometer & $40(16)$ \\
Use a refrigerator temperature & $33(14)$ \\
Wash hands (according to recommendation) & $18(7)$ \\
Wash or clean home kitchen more frequently & $15(6)$ \\
\hline
\end{tabular}

17

18 
Figure

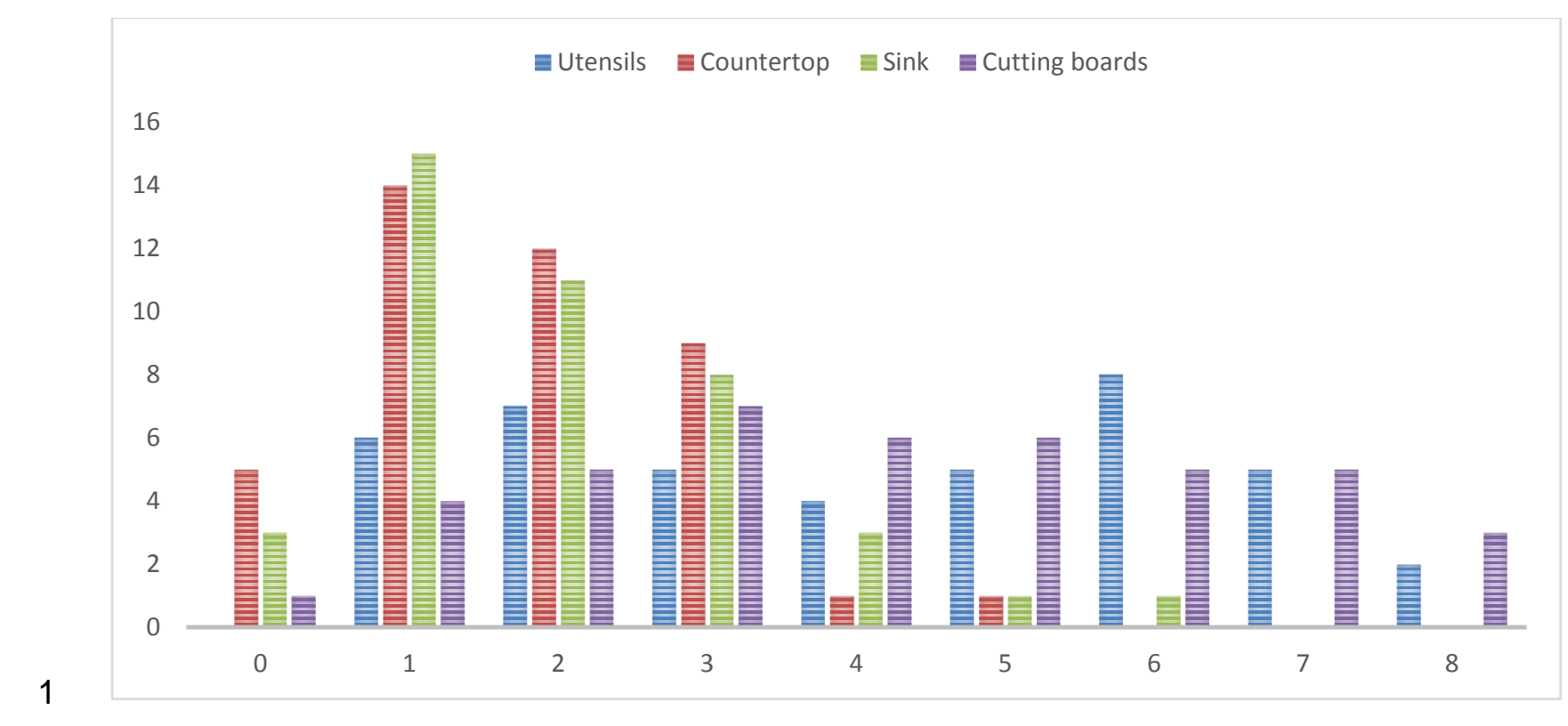

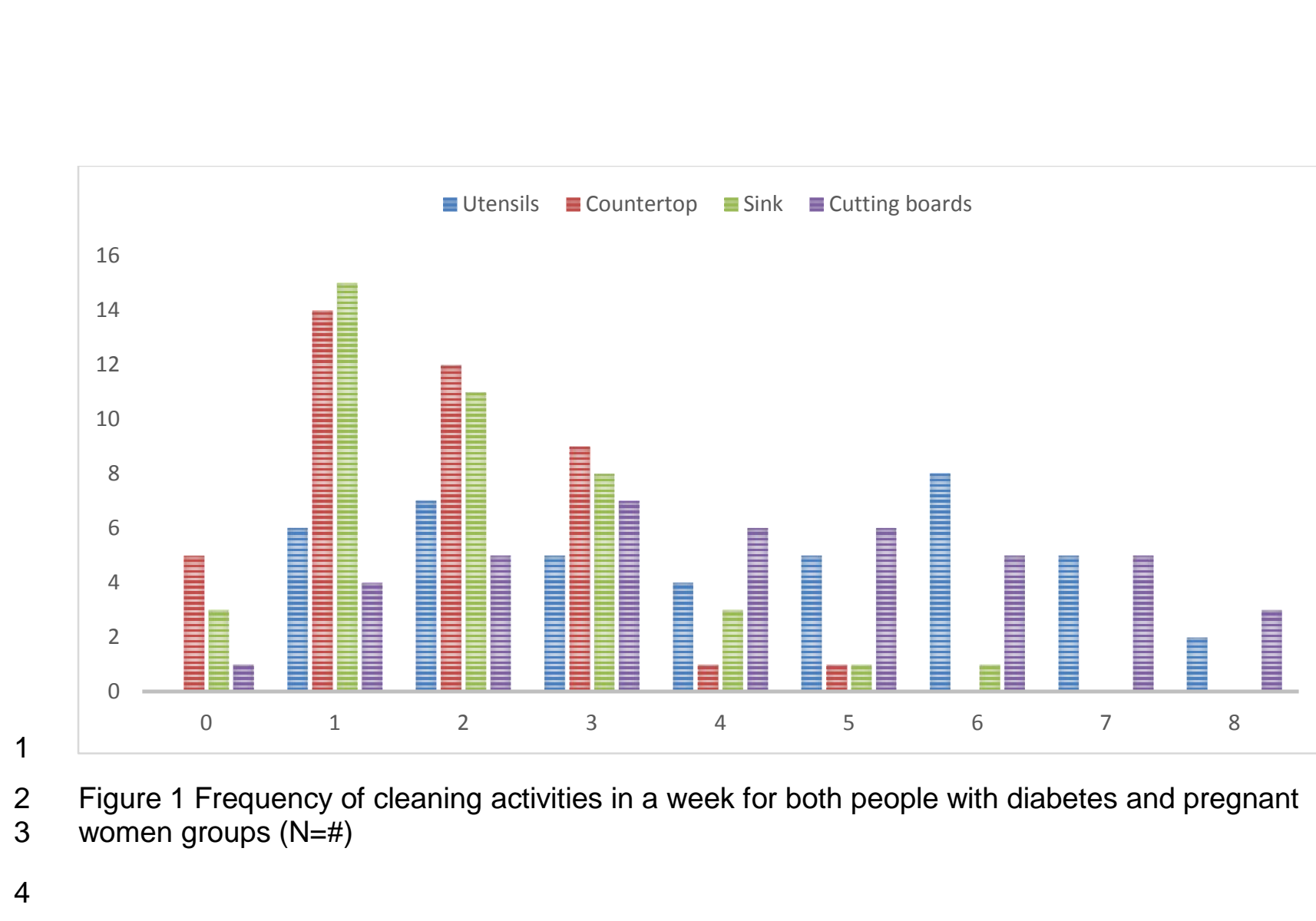
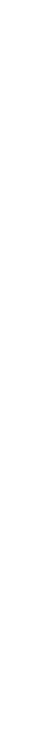

$x^{2}$

(2) 